\title{
Self-repelling fractional Brownian motion - a generalized Edwards model for chain polymers
}

\author{
Jinky Bornales \\ Physics Dept., MSU-IIT, Iligan, The Philippines. \\ E-mail: jinky.bornales@g.msuiit.edu.ph \\ Maria João Oliveira \\ Univ. Aberta and CMAF, University of Lisbon. \\ E-mail: oliveira@cii.fc.ul.pt \\ Ludwig Streit \\ BiBoS, Univ. Bielefeld and CCM, Univ. da Madeira \\ e-mail: streit@uma.pt
}

April 18, 2019

\begin{abstract}
We present an extension of the Edwards model for conformations of individual chain molecules in solvents in terms of fractional Brownian motion, and discuss the excluded volume effect on the end-to-end length of such trajectories or molecules.
\end{abstract}

\section{Introduction}

Individual chain polymers in good solvents are typically modelled by trajectories of random walks, or - in the continuum limit - by Brownian paths. Such models by themselves however do not take into account that self-crossings of these paths should be suppressed, this "the excluded volume" effect will 
make the trajectories less curly and more extended. Fractional Brownian paths have been suggested as a heuristic model for such swelling, or on the other hand for polymers in a collapsed state [2], but a more proper model would be based on self-avoiding random walks, or on a weight factor which penalizes self-crossings, such as in the continuum Edwards [3] [6] [20] [21] [22] 23] or the discrete Domb-Joyce [5] model.

The ensuing swelling of the molecular conformations is given by the Flory index [8] [9] which describes the scaling of the end-to-end distance as a function the number of monomers. It has been extensively studied both in the (chemical) physics and the mathematics community. The physics literature is characterized by structural intuition and far-reaching predictions, the mathematical results are less far-reaching but provide the high reliability characteristic of the mathematical approach. Both are too vast to be quoted here, we refer for this to recent reviews [12] [18].

In the present paper, after a few words on fractional Brownian motion $\mathrm{fBm}$, we shall see that one can extend to $\mathrm{fBm}$ the Edwards model of Brownian paths with exponentially suppressed self-intersections, a mathematical existence proof has been established recently [11. In the third part of the paper we generalize some by now classical arguments from the physics literature to explore what the Flory index might be in the fBm case.

\section{The fBm Edwards Model}

\subsection{Fractional Brownian Motion}

Fractional Brownian motion on $\mathbb{R}^{d}, d \geq 1$, with "Hurst parameter" $H \in(0,1)$ is a $d$-dimensional centered Gaussian process $B^{H}=\left\{B^{H}(t): t \geq 0\right\}$ with covariance function

$$
\mathbb{E}\left(B_{i}^{H}(t) B_{j}^{H}(s)\right)=\frac{\delta_{i j}}{2}\left(t^{2 H}+s^{2 H}-|t-s|^{2 H}\right), \quad i, j=1, \ldots, d, s, t \geq 0 .
$$

For $H=1 / 2$ it is ordinary $d$-dimensional Brownian motion $B$. We refer to the recent monographs by Biagini et al. [1] and by Y. Mishura [16]; for self-intersection local times of fBm see $\mathrm{Hu}$ and Nualart [13]. 


\subsection{The Edwards Model}

Self-repelling Brownian paths for a time interval $0 \leq t \leq l$ can be modelled via a "Gibbs factor" to suppress self-intersections:

$$
G=\frac{1}{Z} \exp \left(-g \int_{0}^{l} d s \int_{0}^{l} d t \delta(B(s)-B(t))\right) .
$$

Technically one defines this expression as a limit, using

$$
\delta_{\varepsilon}(x):=\frac{1}{(2 \pi \varepsilon)^{d / 2}} e^{-\frac{|x|^{2}}{2 \varepsilon}}, \quad \varepsilon>0
$$

in particular

$$
Z=\lim _{\varepsilon \rightarrow+0} \mathbb{E}\left(\exp \left(-g \int_{0}^{l} d s \int_{0}^{l} d t \delta_{\varepsilon}(B(s)-B(t))\right)\right)
$$

if this quantity is well defined; otherwise a renormalization is required, as, more generally, in Theorem 2.2 below.

Recently, generalizing an argument of Varadhan [20], this was extended in [11] to

$$
G=\frac{1}{Z} \exp \left(-g \int_{0}^{l} d s \int_{0}^{l} d t \delta\left(B^{H}(s)-B^{H}(t)\right)\right)
$$

as follows.

Theorem 1 The Edwards model is well defined for all $H<1 / d$, with

$$
G=\frac{1}{Z} \exp \left(-g \int_{0}^{l} d s \int_{0}^{l} d t \delta\left(B^{H}(s)-B^{H}(t)\right)\right) .
$$

Theorem 2 For $H=1 / d$ and $g$ sufficiently small

$$
G=\lim _{\varepsilon \searrow 0} \frac{1}{Z_{\varepsilon}} \exp \left(-g \int_{0}^{l} d s \int_{0}^{l} d t \delta_{\varepsilon}\left(B^{H}(s)-B^{H}(t)\right)\right),
$$

with

$$
Z_{\varepsilon} \equiv \mathbb{E}\left(\exp \left(-g \int_{0}^{l} d s \int_{0}^{l} d t \delta_{\varepsilon}\left(B^{H}(s)-B^{H}(t)\right)\right)\right)
$$

is well-defined. 


\section{The Flory Index}

When the number $N$ of monomers of a polymer becomes large one expects its end-to-end length $R$ to scale [10]

$$
R(N) \sim N^{v}
$$

For (fractional) Brownian motion the root-mean-square length

$$
R=\sqrt{\mathbb{E}\left(B^{H}(N)^{2}\right)}
$$

is scaling with

$$
v=H \text {. }
$$

But the excluded volume effect makes the paths and polymers swell: the end-to-end length increases. For the Brownian motion case there is the famous Flory formula

$$
v=v(d)=\frac{3}{d+2}
$$

based originally on a mean field argument. Since its proposal by Flory [7] [9], numerous methods were invoked to put it on a more solid mathematical basis, a process which has up to now been fully successful in the case $d=1$ [12].

To obtain what may be considered as a first guess of a similar formula for fBm we shall return to the modest beginnings, generalizing Fisher's original argument [7] [8] (see e.g. the review given in McKenzie [15]) to the case at hand.

\subsection{The Fisher Argument}

A partition function $Z(R)$ for a freely jointed chain of $N$ segments for which the end-to-end length has fixed modulus $R$ is given by

$$
Z(R)=a R^{d-1} \exp \left(-\frac{d R^{2}}{2 N}\right)
$$

and leads to a free energy

$$
F_{1}=-\ln Z \sim \frac{d R^{2}}{2 N}-(d-1) \ln R
$$


Instead of such a chain a continuous model is that of a Brownian trajectory from time zero to time $N$, for which one computes

$$
\mathbb{E}(\delta(B(N)-\vec{R}))=(2 \pi N)^{-d / 2} \exp \left(-\frac{R^{2}}{2 N}\right) .
$$

For the $\mathrm{fBm}$ case this formula generalizes to

$$
\mathbb{E}\left(\delta\left(B^{H}(N)-\vec{R}\right)\right)=\left(2 \pi N^{2 H}\right)^{-d / 2} \exp \left(-\frac{R^{2}}{2 N^{2 H}}\right)
$$

from which we see that $N \rightarrow N^{2 H}$, and hence we should consider

$$
Z(R)=a R^{d-1} \exp \left(-\frac{d R^{2}}{2 N^{2 H}}\right)
$$

i.e.

$$
F_{1}=-\ln Z \sim \frac{d R^{2}}{2 N^{2 H}}-(d-1) \ln R
$$

For the repulsive excluded volume energy of fBm paths $x$ with $x(N)=\vec{R}$,

$$
F_{2}=-\ln \mathbb{E}_{x(N)=\vec{R}}\left(\exp \left(-g \int_{0}^{N} d s \int_{0}^{N} d t \delta(x(s)-x(t))\right)\right)
$$

dimensional considerations and mean field arguments [15] suggest

$$
F_{2} \sim \operatorname{const} \cdot \frac{N^{2}}{R^{d}}
$$

Maximizing

$$
F(N, R)=F_{1}(N, R)+F_{2}(N, R)
$$

with regard to $R$ leads to

$$
0=\frac{d R}{N^{2 H}}-\frac{d-1}{R}-\text { const. } N^{2} R^{-d-1} .
$$

Assuming that the 2 nd term is negligible one finds

$$
R^{d+2} \sim N^{2 H+2}
$$

i.e.

$$
R \sim N^{v_{H}}
$$

with

$$
v_{H}(d)=\frac{2 H+2}{d+2}
$$


Remark 3 A polymer model with $B\left(t^{2 H}\right)$ instead of $B^{H}(t)$ would produce the same expression as in (2), hence also the same Flory index, but would not share the homogeneity implied by the stationary increments of $B^{H}(\cdot)$.

\subsection{The Critical Dimension}

The derivation of $v_{H}$ is evidently heuristic and needs validation. For this it is worth noting that for Brownian motion there is a critical dimension $d_{c}=4$ defined by the fact that for $d \geq d_{c}$ there is no excluded volume effect, so that $R$ scales like the unperturbed Brownian motion:

$$
R \sim N^{1 / 2}
$$

i.e.

$$
v_{1 / 2}(4)=1 / 2 \text {. }
$$

We can ask for which dimension, more generally, the fBm Flory index will show no excluded volume effect from self-crossings, i.e.

$$
v_{H}\left(d_{c}\right)=H .
$$

Inserting our ansatz (3) one finds

$$
H d_{c}=2 .
$$

and indeed it is known (Theorem 1.1 of Talagrand [19]) that $d$-dimensional fBm has no double points iff

$$
H d \geq 2,
$$

in other words, our $v_{H}$ predicts $d_{c}$ correctly.

Remark 4 As a consequence, any Flory formula should be considered only up to the critical dimension, i.e. as long as there are double points and an excluded volume effect. Similarly, any prediction of $v_{H}>1$ would be unphysical: the end-to-end distance cannot grow faster than the number $N$ of monomers. In the case at hand this suggests for the one-dimensional case

$$
v_{H}(1)=\left\{\begin{array}{cl}
\frac{2 H+2}{3} & \text { if } H \leq \frac{1}{2} \\
1 & \text { if } H>\frac{1}{2}
\end{array} .\right.
$$


Note that for small $H$ the scaling exponent as predicted would be strictly less than one while for the Brownian motion case

$$
v_{1 / 2}(1)=1
$$

has been proven [12] [23]. The infimum

$$
\lim _{H \rightarrow 0} v_{H}(1)=2 / 3
$$

happens to be the scaling exponent of the myopic random walk [12].

Remark 5 In the attached figure 1 the two red lines correspond to $v_{H}(d)=1$ and to the critical dimension as a function of the Hurst index $H$, respectively. Above these the Flory index is unphysical. On the green lines $v_{H}$ is validated. (The existence proof of the fBm Edwards model in [11] works below the dashed line.)

Remark 6 For fixed dimension d, any extension $F(H)$ of the Flory formula to general Hurst indices $H$ will have to obey

$$
F\left(\frac{1}{2}\right)=\frac{3}{d+2}
$$

for the usual Brownian motion (Flory-Fisher), and

$$
F\left(\frac{2}{d}\right)=\frac{2}{d}
$$

at the critical point (Talagrand). Note that our ansatz (3)

$$
F(H)=v_{H}(d)=\frac{2 H+2}{d+2}
$$

is just the unique linear interpolation between those two values.

\subsection{A Recursion Formula}

For $H=1 / 2$ Kosmas and Freed [14] derive a recursion formula

$$
2-\frac{1}{v(d)}=\frac{4-d}{3}\left(2-\frac{1}{v(1)}\right)
$$


(Here and in the following we label formulas from - or analogous to those in - the paper [14 by their numbers in that article, in double brackets.)

The derivation of this formula is specific to the Brownian motion case and does not hold for general $v_{H}$. Hence in what follows we shall generalize their arguments which led to (4.13) to first obtain a valid recursion formula and then check whether it is satisfied by $v_{H}$ as given in (3).

We begin by considering

$$
Z(g, N) \equiv \mathbb{E}\left(\exp \left(-g \int_{0}^{N} d s \int_{0}^{N} d t \delta\left(B^{H}(s)-B^{H}(t)\right)\right)\right) .
$$

From the defining relation

$$
\mathbb{E}\left(B^{H}(s) B^{H}(t)\right)=\frac{1}{2}\left(s^{2 H}+t^{2 H}-|s-t|^{2 H}\right)
$$

we see that for $a>0$ the processes $\left\{B^{H}(t): t>0\right\}$ and $\left\{a^{-H} B^{H}(a t): t>0\right\}$ obey the same law. Making this substitution and a change of integration variables as $=\sigma$, at $=\tau$ we obtain

$$
\begin{aligned}
Z(g, N) & =\mathbb{E}\left(\exp \left(-g a^{H d-2} \int_{0}^{a N} d s \int_{0}^{a N} d t \delta\left(B^{H}(s)-B^{H}(t)\right)\right)\right) \\
& =Z\left(a^{H d-2} g, a N\right) .
\end{aligned}
$$

Likewise we find for the mean-square end-to-end distance

$$
\begin{aligned}
\left\langle R^{2}\right\rangle & \equiv \frac{1}{Z(g, N)} \mathbb{E}\left(\left(B^{H}(N)\right)^{2} \exp \left(-g \int_{0}^{N} d s \int_{0}^{N} d t \delta\left(B^{H}(s)-B^{H}(t)\right)\right)\right) \\
\left\langle R^{2}\right\rangle & =a^{-2 H} \mathbb{E}\left(\left(B^{H}(a N)\right)^{2} \exp \left(-g a^{H d-2} \int_{0}^{a N} d s \int_{0}^{a N} d t \delta\left(B^{H}(s)-B^{H}(t)\right)\right)\right) \\
& =a^{-2 H} f\left(a^{H d-2} g, a N\right) \\
& =N^{2 H} f\left(N^{2-H d} g, 1\right) .
\end{aligned}
$$

(Note the critical dimension $H d=2$ where $\left\langle R^{2}\right\rangle^{1 / 2} \sim N^{H}$.) For large $N$ one expects a power law behavior for the unknown function $f$, i.e.

$$
\left\langle R^{2}\right\rangle \sim N^{2 H}\left(N^{2-H d} g\right)^{x}
$$


with an exponent $x$ to be determined.

As a next step we restrict one coordinate of the positions, $x_{i}(t)=B_{i}^{H}(t)$ to the interval $[0, D]$ by inserting

$$
1_{[0, D]}\left(B_{i}^{H}\right)=\left\{\begin{array}{cc}
1 & \text { if } B_{i}^{H}(t) \in[0, D] \text { for all } t \\
0 & \text { otherwise }
\end{array}\right.
$$

into (6). One obtains

$$
\begin{gathered}
\left\langle R^{2}\right\rangle_{D}=\frac{1}{Z_{D}(g, N)} \mathbb{E}\left(1_{[0, D]}\left(B_{i}^{H}\right)\left(B^{H}(N)\right)^{2} \exp \left(-g \int_{0}^{N} d s \int_{0}^{N} d t \delta\left(B^{H}(s)-B^{H}(t)\right)\right)\right) \\
=\frac{a^{-2 H}}{Z_{D}(g, N)} \mathbb{E}\left(1_{\left[0, a^{H} D\right]}\left(B_{i}^{H}\right)\left(B^{H}(a N)\right)^{2} \exp \left(-a^{H d-2} g \int_{0}^{a N} d \sigma \int_{0}^{a N} d \tau \delta\left(B^{H}(\sigma)-B^{H}(\tau)\right)\right)\right) \\
=a^{-2 H} F\left(a^{H} D, a^{H d-2} g, a N\right)=N^{2 H} F\left(N^{-H} D, N^{2-H d} g, 1\right) .
\end{gathered}
$$

Now assume that asymptotically there is a dimensionless correction factor $h$ for

$$
\left\langle R^{2}\right\rangle_{D} \approx\left\langle R^{2}\right\rangle h\left(\frac{D}{\sqrt{\left\langle R^{2}\right\rangle}}\right) .
$$

It should grow as $D$ becomes small which suggests a power law behavior for the function $h$ :

$$
\left\langle R^{2}\right\rangle_{D} \approx\left\langle R^{2}\right\rangle\left(\frac{D}{\sqrt{\left\langle R^{2}\right\rangle}}\right)^{-y}
$$

with $y$ to be determined. As $D$ approaches a minimal value $D_{0}$ - approximately the extension of a monomer ("Kuhn length") - the polymer becomes effectively $(d-1)$-dimensional:

$$
\left\langle R_{d-1}^{2}\right\rangle \approx\left\langle R_{d}^{2}\right\rangle_{D} \approx D_{0}^{-y}\left\langle R_{d}^{2}\right\rangle^{1+\frac{y}{2}}
$$

This provides a relation between the end-to-end length for dimensions $d$ and $d-1$. To obtain from this a recursion relation, recall equation $(2.22)]$ :

$$
\left\langle R_{d}^{2}\right\rangle \sim N^{2 H+x(2-H d)} g^{x}
$$

and introduce instead of $x$ the (unknown)

$$
2 v_{H}(d) \equiv 2 H+x(2-H d)
$$


so that

$$
\left\langle R_{d}^{2}\right\rangle=c_{d} N^{2 v_{H}(d)} g^{\frac{2 v_{H}(d)-2 H}{2-H d}}
$$

and

$$
\left\langle R_{d-1}^{2}\right\rangle=c_{d-1} N^{2 v_{H}(d-1)} g^{\frac{2 v_{H}(d-1)-2 H}{2-H \cdot(d-1)}} .
$$

On the other hand from $(4.6)$ we have

$$
\left\langle R_{d-1}^{2}\right\rangle \approx D_{0}^{-y}\left\langle R_{d}^{2}\right\rangle^{1+\frac{y}{2}}=\text { const. } N^{2 v_{H}(d)\left(1+\frac{y}{2}\right)} g^{\frac{2 v_{H}(d)-2 H}{2-H d}\left(1+\frac{y}{2}\right)} .
$$

Comparing exponents in these two expressions we find

$$
\begin{aligned}
v_{H}(d-1) & =v_{H}(d)\left(1+\frac{y}{2}\right) \\
\frac{v_{H}(d-1)-H}{2-H \cdot(d-1)} & =\frac{v_{H}(d)-H}{2-H d}\left(1+\frac{y}{2}\right) .
\end{aligned}
$$

The first of these equations gives

$$
1+\frac{y}{2}=\frac{v_{H}(d-1)}{v_{H}(d)},
$$

with this the second one becomes

$$
\frac{1}{v_{H}(d-1)} \frac{v_{H}(d-1)-H}{2-H \cdot(d-1)}=\frac{1}{v_{H}(d)} \frac{v_{H}(d)-H}{2-H d}
$$

i.e. this expression does not depend on the dimension $d$ so that all the $v_{H}(d)$ are given in terms of e.g. $v_{H}(1)$ :

$$
\begin{aligned}
& \frac{1}{v_{H}(d)} \frac{v_{H}(d)-H}{2-H d}=\frac{1}{v_{H}(1)} \frac{v_{H}(1)-H}{2-H}, \\
& \Longrightarrow v_{H}(d)=\frac{(2-H) v_{H}(1)}{(d-1) v_{H}(1)+2-d H} .
\end{aligned}
$$

\section{Proposition 7}

$$
v_{H}(d)=\frac{2 H+2}{d+2}
$$

satisfies this recursion equation, with

$$
\frac{1}{v_{H}(d)} \frac{v_{H}(d)-H}{2-H d}=\frac{1}{2 H+2} .
$$


Remark 8 The standard Flory index (3) for $H=1 / 2$ obeys the recursion formula.

Remark 9 The recursion formula (8) implies the correct critical behavior, i.e. any solution will obey $v_{H}(d)=H$ for $d=2 / H \equiv d_{c}$, whatever the choice of $v_{H}(1)$. To see this explicitly, insert $d=2 / H$ and find

$$
v_{H}\left(\frac{2}{H}\right)=\frac{(2-H) v_{H}(1)}{(2 / H-1) v_{H}(1)+0}=H .
$$

Remark 10 If $v_{H}(1)$ turned out to be equal to one for all $H$, the recursion formula would suggest

$$
v_{H}(d)=\frac{2-H}{d+1-d H}
$$

an expression which then also produces the standard Flory formula for $H=$ $1 / 2$, as well as the critical dimension $d=2 / H$.

\section{Summary}

The Edwards type model for self-repelling fBm now at hand will raise the question of how the end-to-end length of trajectories scales as a function of time (or "number of monomers"). The original Fisher argument, while criticized regarding its assumptions [17] [4, provides a simple heuristic "derivation" of the Flory formula which allows an extension to fBm. The obtained scaling law needs further verification; we note that it correctly predicts the critical dimension for which the excluded volume become negligible and obeys a recursion formula based on dimension reduction. The latter would provide a useful constraint on any alternate scaling laws.

$$
* * *
$$

J. B. and L. S. would like to express their gratitude for the kind hospitality of the Centro de Matemática e Aplicações Fundamentais CMAF in Lisbon which made our collaboration possible and pleasant. This work is supported by PTDC/MAT/100983/2008, ISFL-1-209. 


\section{References}

[1] F. Biagini, Y. Hu, B. Oksendal: Stochastic Calculus for Fractional Brownian Motion and Applications. Springer, Berlin, 2007.

[2] P. Biswas, B. J. Cherayil: Dynamics of Fractional Brownian Walks. J. Phys. Chem. 99, 816-821 (1995).

[3] E. Bolthausen: On the construction of the three dimensional polymer measure. Prob. Theory. Rel. Fields 97, 81-101 (1993).

[4] J. Des Cloizeaux: On the absence of flory terms in the energy and in the entropy of a polymer chain. J. Phys. France 37, 431-434 (1976).

[5] C. Domb, G. S. Joyce: Cluster expansion for a polymer chain. J. Physics C 5, 956-976 (1972).

[6] S. F. Edwards: The statistical mechanics of polymers with excluded volume. Proc. Phys. Sci. 85 613-624 (1965)

[7] M. E. Fisher: Shape of a self-avoiding walk or polymer chain, J. Chem. Phys. 44, 616-622 (1966).

[8] M. E. Fisher: J. Phys. Soc. Japan 26 Suppl. 44 (1969).

[9] P.J. Flory: Principles of Polymer Chemistry. Cornell University Press, 1953.

[10] P. G. de Gennes: Scaling Concepts in Polymer Physics. Cornell University Press, Ithaca, NY, 1979.

[11] M. Grothaus, M.J. Oliveira, J.-L. Silva, L. Streit.: Self-avoiding fractional Brownian motion - The Edwards model. J. Stat. Phys. 145 (6), 1513-1523 (2011).

[12] R. van der Hofstad, W. König: A survey of one-dimensional random polymers. J. Stat. Phys. 103, 915-944 (2001).

[13] Y. Hu and D. Nualart: Renormalized self-intersection local time for fractional Brownian motion. Ann. Probab. 33, 948-983 (2005)

[14] M. K. Kosmas, K. F. Freed: On scaling theories of polymer solutions. J. Chem. Phys. 69, 3647-3659 (1978). 
[15] D. S. McKenzie: Polymers and Scaling. Phys. Rep. 27, 35-88 (1976).

[16] Y. Mishura: Stochastic Calculus for Fractional Brownian Motion and Related Processes. Springer LNM 1929, 2008.

[17] M. A. Moore, A. J. Bray: On the Flory formula for the polymer size exponent v. ,J. Phys. A: Math. Gen. 11, 1353-1359 (1978).

[18] A. Pelissetto, E. Vicari: Critical phenomena and renormalization-group theory. Phys. Rep. 368, 549-727 (2002), Chapter 9.

[19] M. Talagrand: Multiple points of trajectories of multiparameter fractional Brownian motion. Probab. Theory Related Fields 112, 545-563 (1998).

[20] S. R. S. Varadhan: Appendix to "Euclidean quantum field theory" by K. Symanzik, in: R. Jost, ed., Local Quantum Theory, Academic Press, New York, p. 285, 1970.

[21] J. Westwater: On Edwards' model for polymer chains. Comm. Math. Phys. 72, 131-174 (1980).

[22] J. Westwater: On Edwards' model for polymer chains. III. Borel summability. Comm. Math. Phys. 84, 459-470 (1982).

[23] J. Westwater: On Edwards model for polymer chains, in Trends and Developments in the Eighties, S. Albeverio and P. Blanchard, eds., Bielefeld Encounters in Math. Phys. 4/5 (World Scientific, Singapore, 1984). 


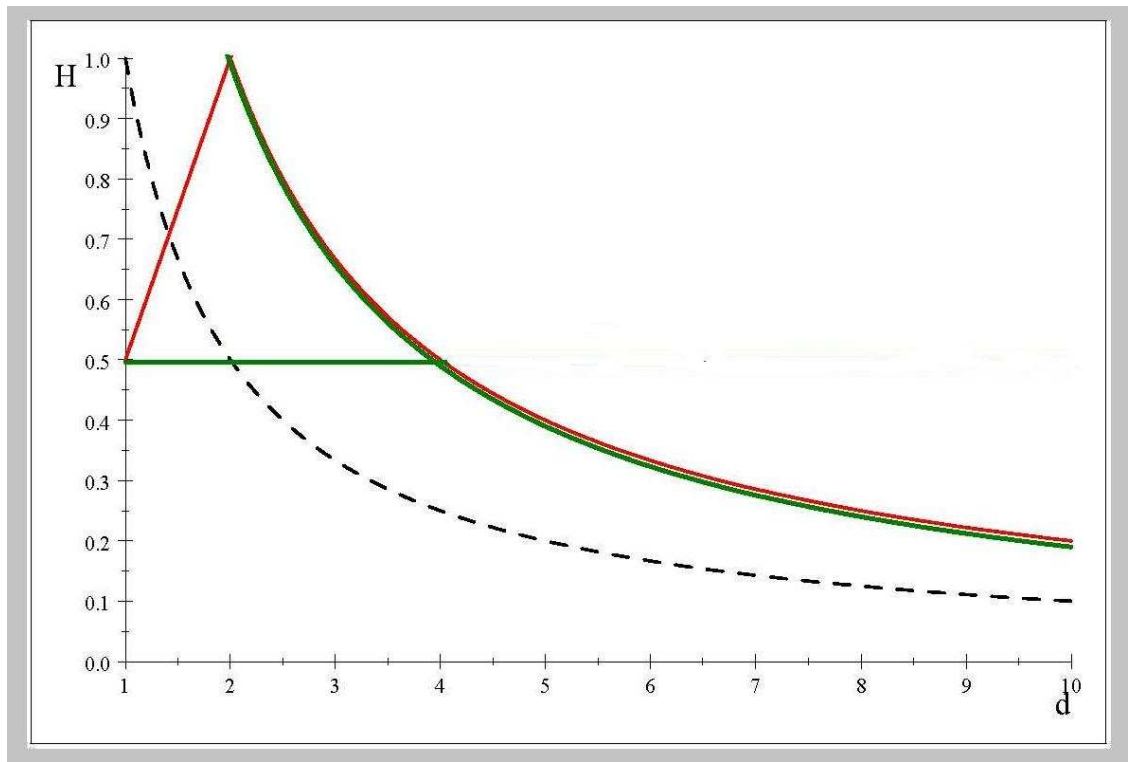

Figure 1: The domain of the Flory index 